\title{
DER FRONTSOLDAT SIEHT SICH SELBST UND DIE FEINDE - EIN KURZABRISS
}

Die Wurzel des Kriegsdenkens ist der Grundgedanke, sich selbst zu erhalten und den Feind zu eliminieren. Der Einzelkämpfer ist auf dem Kampffeld für sich ein Stratege. Und das ist eine positivistische, eine pragmatische Haltung. Jegliche rationale Diskussion ist ausgeschlossen. Diejenigen Wörter, Ideen, die zum Etablierten nicht passen, bleiben außerhalb der Gesellschaft. Selbst falls andere Wörter und Meinungen ausgesprochen und gehört werden, sind sie nur im Sinn der offiziellen Sprache verstanden. Fremde Völker werden dann als fremde Arten behandelt, wobei blinde Leidenschaften und rationale Absichten unzertrennbar erscheinen. Die eigene Seite bleibt dabei immer rational. Am Rande der Geschehnisse befindet sich die nicht zahlreiche, aber sehr aktive Gruppe der Abenteurer, die bei Kriegsereignissen immer da waren.

Entsteht ein Konfliktfeld, so werden alle Teilnehmer von Erregung erfasst, als Tätige oder Opfer. Partei ergreifen heißt dann - Soldat zu werden. Nur wenige ziehen aber in den Krieg als bewusste Teilnehmer - die Mehrheit realisiert wohl ihre unterdrückten Aggressionen und Selbstzerstörungsdränge. „Kampfterror“ verursacht erbarmungslose Grausamkeit, die keine kulturbedingte Begründung braucht und den Feind aller Menschenwürde beraubt. Die blinden Leidenschaften werden taktisch als rationale Überlegungen ausgegeben, dagegen werden echte rationale Überlegungen von der Gegenseite als Ausdruck blinden Hasses dargestellt. Die Masse bleibt in diesen Hetzkampagnen ohne Instrumente, womit sie sich objektive Informationen verschaffen könnte.

Die Erfahrung, dass die Kriege immer fast unerwartet kommen, zeigt, wie notwendig es ist, dieses grausame und heimtückische Geschehen zu begreifen.

Das Thema des Krieges ist auch in kleinen Aspekten so umfangreich, dass man gezwungen ist, eigentlich nur Bruchteile der Kriegswirklichkeit zu untersuchen. Und diese sind mit den anderen, ähnlichen so eng verbunden, dass die Analyse mehr oder weniger oberflächlich bleibt, und weil die Zeugen nicht mehr da sind, unterliegen unsere Meinungen den neuen Moden und Tendenzen. Nur eine

* Dr. Anatol Kapphengst, Universität Gdańsk, Institut für Germanistik, ul. Wita Stwosza 51, 80-308 Gdańsk. E-mail: alphart@interia.pl 
Tatsache bleibt wohl so, wie sie auch damals war, die wahre oder nur empfundene Ohnmacht von allen damaligen Bewohnern Europas, so wie es Golo Mann vor Jahren über die Verhaltensweise des deutschen Kaisers sagt: „... getrieben von den Generälen, getrieben von einer Volksmeinung, die ihrerseits durch eine demagogische Presse aufgepeitscht worden war"(Mann, Golo, 1914 und 1980, "Die Welt", 30.04.1980).In dieser Abhandlung muss man also bewusst auf viele Tatsachen verzichten, die das Thema betreffen.

Es muss gesagt werden, dass in Bezug auf Dokumente auch eine gewisse Vorsicht geboten ist, und die Gründe für diese bemerkt man schon am Anfang des Großen Krieges: ,... zu diesem Zustand verkürzter Erfahrungen gedeiht nun eine überaus reiche Phantasietätigkeit. Tausend Lügen werden geglaubt und die Lüge wird dadurch zu einer furchtbaren Waffe" (DGK, Lamprecht, Karl, Seelische Erscheinungen des Krieges, S. 211-213). Dazu noch kommen die Dokumente überwiegend von den verschonten Menschen, die - und diese Tatsache wurde schon von den Zeitgenossen damals anerkannt - ,die nicht in die Hauptangriffslinie kamen" (BS, am 28.03.1915, S. 172). Dazu kommt noch eine Erscheinung, die Karl Lamprecht so darstellt: „Auf einer Seite ein unbegrenztes Aufgeben des Ich, Opfermut bis zum äußersten, Einschätzung des Lebens nur mit Rücksicht auf das Ganze, auf der anderen Seite Zeichen des willkürlichsten Egoismus, Grausamkeiten schlimmster Art und noch mehr - Neigung, die Berichte über solche Grausamkeiten zu glauben“" (Lamprecht, Karl, Seelische Erscheinungen des Krieges, in: DGK, S. 209-216). Also alles, was als Forschungsmaterial existiert, hat mehr oder weniger ausdrückliche Spuren der oben genannten Faktoren. Den journalistischen Materialien ${ }^{1}$ gegenüber sei eine besondere Vorsicht geboten und diese Tatsache bemerken schon die Frontsoldaten: „Im übrigen ist das Journalistengerede über den Krieg schrecklich und [...] vielfach im Stil ganz unwürdig der tatsächlichen Vorgänge“ (KAK, S. 104). Oder auf diese Weise: „Dass die Redaktionen so prahlen mögen und hetzen und übertreiben, beschönigen und herabsetzen, fällt uns besonders auf die Nerven" (ebenda, S. 130). Auch nicht alle Feldpostbriefe sind glaubwürdig und verdienen Vertrauen, falls sie durch die Presse an die Leser gelangen: „... die meisten der Feldpostbriefe sind von Leuten geschrieben, die sich einige Kilometer hinter der Front herumbewegen“"(Josenhans, Karl, am 9.11.1914, in: KS, S. 35). ${ }^{2}$ Die Augenzeugen sind dazu ausnahmslos tot, was die Forscher zwingt, mit dem gegebenen Material zu arbeiten, obwohl auch die von den Soldaten geschriebenen Briefe nicht immer in den kämpfenden Truppen ihren

${ }^{1}$ Obwohl die Behörden nur 8 Berichterstatter für den westlichen Kriegsschauplatz zulassen. (W, ohne Seitennummer)

${ }^{2}$ Auch zeitgenössische Forscher handeln manchmal wie die damaligen Journalisten, wie z.B. Adam Hochschild in seinem Buch Der Große Krieg, wobei der Autor in Sankt Petersburg 1914 die Barrikaden entdeckt (S. 133) und in Sibirien vor dem Großen Krieg - „Lager“ (S. 387). Vgl., Hochschild, Adam, Der Große Krieg, Stuttgart, 2013. 
Ursprung haben. Noch zu erwähnen ist, dass die Mehrheit der Schreibenden aus den gebildeten Schichten stammt: „... waren es doch vor allem Jugendliche aus dem Bürgertum, aus besonders gebildeten Schichten und vornehmlich aus den Städten, die sich frühzeitig für den Kriegsdienst meldeten“ (DEW, S. 159). Diese haben auch viel mehr Veranlagung und Vorbereitung, um das Erlebte zu beschreiben.

Die Bilder der Kriegsteilnehmer, ihr Verhalten, sind in dieser Abhandlung nur zu vier Bereichen begrenzt - das Eigenbild der kämpfenden Deutschen und die Bilder der Feinde: Franzosen, Russen und Engländer, die meist in den Materialien auftreten. ${ }^{3}$

Versuchen wir also, das Eigenbild des Frontsoldaten und seine Art und Weise den Krieg und die Feinde zu sehen, kennenzulernen.

Fremde Meinungen zum Thema sieht der Frontsoldat mit skeptischem Auge und sein Skeptizismus betrifft viele Quellen. Kriegslyriker sind für ihn „kranke Leute" (KAK, II, S. 73), die Soldaten die nicht unmittelbar etwas erlebt haben - die Autoren von bombastischen Briefen nach Hause (Vgl., Miese Fritz, am 24.12.1914, in: KS, S. 89) und weil man weiß, dass die Emotionen die kämpfenden Soldaten zu stark beeinflussen, mistraute man den Überlebenden (vgl., Ratenhuber, A.H., in KF III, S. 4-5). Besonders die im Hinterland verbleibenden Soldaten phantasieren zum Kriegsthema (Vgl., Petersen, Knut, am 25.10.1914, in KS, S. 109) und verfallen in die Fehler, ,einzelne Fälle und Momente als typisch zu betrachten." (Student, am 29.05.1915, in: KS, S. 154)

Der Frontsoldat beurteilt vor allem den Krieg als Erscheinung und sucht nach Kriegsursachen. Dann sieht man z.B. in den belletrisierten Beschreibungen des Krieges ${ }^{4}$ die Ursache des Krieges in dem Phänomen des Zerfalls der christlichen Religion in die nationalen Konfessionen (vgl., O, S. 41), oder man betrachtet den Krieg als eine Art Strafe, die persönlich konkreten Soldaten betrifft (vgl., BL, S. 35). Es gibt idealisierte Kriegsgründe: „Für Dichtung, Kunst, Philosophie, Kultur, geht es ja in den Kampf..." (Ph.Witkop, Zwei Frühgefallenen, Bavin, 18.11.1914, in DGK, S. 76) und wenn deutsche Soldaten zufälligerweise nicht kämpfen, so räumen sie eine Stadt (vgl., JK, S. 103-104). Manche Urteile sind humorvoll: Die Leute haben ja den Krieg gerne, wenn der verfluchte Heldentod nicht wäre." (KAK, Leutnant G., S. 73). Der Krieg an sich betrachtet man im Graben als etwas Schreckliches, Barbarisches, was Unheil bringt. (vgl., Franke, Fritz, am 5.11.1914, in: KS, S. 91; Steinbrecher, H.G., am 3.11.1914, ebenda, S. 292).

${ }^{3}$ Es muss auch ein Dokument erwähnt werden, das das Leben im Graben reglementiert; in welchem als Norm Sturmangriff ohne Beachtung der Verluste, ohne Beachtung der Gegenwirkung des Feindes, mit Endphase ,Nahkampf' befohlen wird. Vgl., Exerzierreglement für die Infanterie, 29.05.1913, Neuabdruck mit Einfügung der bis April 1913 ergangenen Änderungen, Berlin 1913, Punkte 324, 325, 345.

${ }^{4} \mathrm{Zu}$ diesen gehören: die jedoch eindeutig die Kriegserlebnisse der Autoren darstellen. 
Man mildert dabei die eigene Rolle im Kriegsgeschehen, indem man im Kampf „eine blutige [...] Arbeit" sieht. ( Hopp, A., am 1.03.1915, in: KS, S. 42) und die deutschen Soldaten an der Grausamkeit des Krieges ,wahrlich nicht schuld“ (MF, S. 78) sind. Man macht diese Arbeit auch mit Begeisterung, ohne die Resultate zu beschreiben (vgl., Passow, A., in: KF, S. 77). Ob also in dieser Situation zahlreiche Soldaten am Anfang des Krieges Sehnsucht nach Frieden verspüren, wie ein Student am 7. November 1914 schreibt (vgl., Student, S. 21), bleibt ungewiss. Man verwünscht den Krieg, aber jegliche Gedanken, das Gewehr fortzuwerfen, ist den Soldaten fremd. (vgl., Lebenserinnerungen des Schriftsetzers Felix Fronrobert, Privbes., 1979, in: FA, S. 35) Den Feind betrachtet man als ebenbürtig (vgl., Pöhlmann, M., Der Erste, S. 149), aber ebenso oft entmenschlicht man den Gegner, indem man aus ihm Figuren macht (vgl., DK II, aus Wiesbadener Tageblatt, S. 254), was die Tötungsbegeisterung anheizt: „Schießen und treffen mitten hinein in das tückische schwarze Feindesherz.“ (Fuchs Wehner, im November 1914, in: KF, S. 21) Einige Soldaten entwickeln in sich schon vor dem Kampf Kriegswut: „Man trank ,Auf den ersten Russen!'Die sollen sehen, was deutsche Hiebe sind, die Hunde!“ (K, S. 9). Danach behandelt man gelassen nicht nur Russen (vgl., DK, S. 176), sonder alle gnadenlos, man erschießt verwundete Feinde (vgl., K., S. 60), die Sturmtruppen sogar keinen Engländer gefangen, was dem Kriegsrecht widerspricht, man beraubt die Gefangenen (vgl., Feuer, S. 163, 168), beziehungsweise erschießt man bei Fluchtversuch einen französischen Oberleutnant. (vgl., Ratenbacher, A.H., in: KF, III, S. 4) Die Urteile sind kalt und objektiv - so bezweifelt der Soldat die Tatsache, ob die Franzosen etwas für konfiszierte Schlachttiere bekommen. (vgl., Passow, A., in: KF, II, S. 21) $)^{5}$ Die antihumane Betrachtung des Gegners bedeutet jedoch nicht, dass man die eigenen Soldaten als wertvolle Schöpfungen betrachtet, denn sie haben meist weniger Wert als ihre Ausrüstung. Man empfiehlt also, den Verwundeten nicht zu helfen (vgl., KAK, S. 87) um die Zeit nicht zu verlieren, dazu noch sind die Verwundeten fast schädlich, weil sie ihre Ausrüstung verlassen: „Viel kostbares Material ging dadurch verloren!“( (MF, S. 87) Vielleicht deshalb benutzt man jede Möglichkeit, materielle Verluste, nicht obligat der militärischen Art, auszugleichen. Man nimmt also Spirituosen, Näschereien und Kleinigkeiten als Beute (vgl., KAK, S. 39), wie auch Brot und Konserven, weil die in der deutschen Armee knapp waren. (vgl., MF, S. 87; Feuer, S. 175) Nebenbei nimmt man sogar den eigenen Gefallenen alles, was man braucht (vgl., Feuer, S. 96). Das entspricht jedoch der Meinung eines Soldaten, dass „der tapfere Soldat etwas vom Verbrecher“ (KAK, S. 69) hat.

Die Verhältnisse im Graben lassen sich am besten so beschreiben: „Ganz vorn gibt es gar keine Unterstände, man hockt im Trichter oder in der Sappe. Der

${ }^{5}$ Einige Beispiele für deutsche Quittungen für konfiszierte Waren: „Diesen Alten eine Tracht Prügel für ihren ausgezeichneten Käse! Dem Überbringer dieses drei Ohrfeigen für seine schönen Eier!“ ( T., November 1915, S. 104) 
Kompanieführerstollen ist vier Meter tief. Der des Bataillons acht. Das Regiment und die Brigade haben zehn Meter über sich. Die Unterstände der Division sind betoniert. Vorn gibt es überhaupt kein Waschwasser. Der Kompanieführer kann sich, wenn er einen findigen Burschen hat, einmal am Tage das Gesicht und die Hände waschen. Das Regiment wäscht sich sogar die Hände, bevor es seine Feldküchensuppe aus den Blechbehältern löffelt. Die Brigade, bevor sie speist. Die Division nimmt in jeder Woche ein Bad." (WBS, S. 353)

Spricht man über die Stimmung im Graben, so sieht man gegensätzliche Meinungen - von der Kriegsstimmung des 30-jährigen Krieges (vgl., Brudern, Paul, am 23.09.1914, KS, S. 12) bis - ,hier gleicht ein Tag dem andern ohne Unterschied." (Student, am 2.03.1915) Nur in einem Aspekt der Wirklichkeit sind zahlreiche Autoren einig, dass die Verpflegung mangelhaft ist (vgl., MK, zwischen 19.10.1914-24.06.1915, S. 19, 24, 96, 101, 109). Manche beschreiben sogar das Sortiment: „Wir leben jetzt nur von Brot, Kartoffeln und russischen Konserven.“ Gebel, am 21.08.1915, in: KF, Heft I, S. 21). Andere stellen die Akzente anders: „Trockenes Brot, keine reine Wäsche, 12 Wochen die Lumpen nicht vom Leib gehabt" (Vom Holzfällen zum Höhlenbär nach „Vorwärts“, Berlin, in DK 2, S. 267). Schmutz und verdorbenes Essen ( vgl., Ratenhuber, A.H. KF III, S. 7, 9) begleiten den Soldaten, der ,spärlich ernährt, mangelhaft bekleidet und beschuht, selten beurlaubt, im Urlaub von Mutlosen entmutigt..." (HC, 1916, S. 59) seine Pflichten erfüllt. Ungeziefer, Nässe und Kälte in engen Unterständen gehören zum Alltag, diese Meinung ist allgemein, bei bekannten und unbekannten Autoren. (Vgl., Georg, Zipfel, Weihnacht in Flandern, Flammende, S. 325; KAK, S. 220; Krull, C., Feldpostbrief vom 29.07.1916, Privbes., in : FA, S. 64) Eine andere Behauptung klingt dann wie Hohn: ,... [Graben] sind geräumige Erdwohnungen geworden, in denen es sich wohnen lässt, so gut wie irgendwo in den Kasernen..." (VD, S. 14)

Herkunftsfragen spielen dabei kaum eine Rolle, obwohl man begründeter weise annehmen darf, das die Mehrheit der Zeugnisse von den erwähnten Gebildeten stammt: „Unter viel Feldgrauen ist immer einer, dem man es ansieht, dass er von der Hochschule weglief, um zu seiner deutschen Heimat zu sagen: „Nimm mich!'“ (LG, S. 117). Dieser Gruppe der Soldaten verdanken wir die meisten Materialien, auch zum äußerem Bild des Soldaten, z.B. aus 1917: „Es ist ein sonderbares Wesen, schweigsam, tagelang mit den äußeren Symptomen der Gleichgültigkeit dem Artilleriefeuer ausgesetzt.“ (WBS, S. 311) Den Kampfstress abreagierend, benimmt sich diese düstere Person manchmal so: „, Der Trommler $\mathrm{sa} ß$ neben dem Pionier und wickelte sich den kalten Schwanz einer Ratte um den Finger, die er soeben getötet hatte.“ (O, S. 108) Man betrachtet in dieser Situation sich selbst und die Kameraden gelassen, als Schachfiguren (vgl., KAK, S. 63), die am Spiel teilnehmen, wobei sie in sich eine kafkaeske Verwandlung erleben: „, Der Frontinstinkt findet in jenem Zustand der Wurschtigkeit seinen besten Nährbogen, der Elan wird zu Geduld, der Mut zur Gelassenheit, die Tapferkeit, gegenüber allen Ereignissen äußerer und innerer Art" (WB, S. 236). Im Graben 
sitzen erschlaffte Greise (Vgl., WBS, S. 221; Feldpostbriefe an den Verband der Bergarbeiter Deutschlands 1914-1918, Brief H. Aufderstrasse vom 14.04.1918, aus B. Arch., R 9350, NR 271, in: FA, S. 68-69; Die Front, Ein Großkampftag in der Sommeschlacht 1916, S. 16). Die Bilder direkt aus dem Kampf sind fragmentarisch und bleiben im Gedächtnis als Bruchteile des Erlebten (Vgl., Die Front, Die erste deutsche Durchbruch 1918, Beginn der Infanterieschlacht der, S. 51; nach Jünger, E., In Stahlgewittern; O, S. 145; PE, S. 182), wohl darum, weil das Erlebte zu grausam ist. Als man einen laufenden kopflosen Feind sieht (BL, S. 115), oder eine Sturmwelle, die wie Wahnsinnige feindliche Linien stürmt (O, S. 171), dann fragt man sich danach: „Was waren wir denn? Menschen? Tiere? Dinge?“ (Goes, G., Pater Rupert, in: Flammende Fronten, S. 275) Und als man überlebt, allein aus einer Menge von Kameraden, so bleibt am Ende der Soldat doch innerlich erschlagen: „Ich sah kein siegestrunkenes Auge, hörte keinen berauschenden Siegesgesang, vernahm keinen lärmenden Freudetrubel..." (LuP, am 29.08.1914, S. 33; vgl. auch Feuer, S. 97) Niemand ist dann vor einem Nervenzusammenbruch sicher (vgl., MK, am 4.05.1915, S. 96) und allmählich entsteht aus den Überlebenden fast ein neuer Menschentypus, den wir auch auf den Fotos sehen können. Diese Menschen sind ausgehungert und abgekämpft, mit scharfen Gesichtszügen, Totenmasken ähnlich (vgl., Feuer, S.16, 22): „, Dieser Soldat des dritten Kriegsjahres, dieser stille, verschmutzte, jeder Freude entwöhnte, und hin und wieder einmal halb kindisch, halb verlegen lächelnde Mensch - er, der auf Heimaturlaub sich kaum zurechtfinden vermag und beinahe erleichtert aufatmet, wenn er erst wieder draußen bei der anderen ist..." (WBS, S. 318). Die Abstumpfung der Sinne erfolgt erstaunlich schnell und der Soldat gewöhnt sich an eine Existenz unter noch Lebendigen und schon Toten (vgl., Ratenhuber, A.H, KF, III, S. 9). Der Urlaub bleibt also bei meisten ein Traum (vgl., MK, am 29.06.1915, S. 112), aber daheim fühlt man sich fremd und verlassen, denn die Welt der Einheimischen versteht nichts von der Frontwirklichkeit, die mit Worten unübertragbar ist (vgl., Briefe, Post und Ansichtskarten von zum Kriegsdienst eingezogenen Archivmitarbeitern aus dem Felde 1914-1919, Senat Kriegsakten, A III Z, 17a, Bd.3 K-V, in: FA, S. 36; auch T., Mai 1915, S. 63).

Im Graben duzen einander alle (vgl., BLF, am 6.12.1914, S. 49), was jedoch nicht bedeutet, dass alle füreinander gleichwertig und gleich wichtig sind. Schon der Regimentsstab bildet einen kleinen Hof, wo die Achselstücke am wichtigsten sind (vgl., HC, am 8.11.1916, S. 96), die höheren Stäbe gehören einer fremden, entmenschlichten Welt, und ein Grabenoffizier ,hätte sie alle töten können“ (T., November 1915, S. 106).Kameradschaftliche Gefühle enden auf dem Niveau der Kompanieführer, die - falls sie länger am Leben bleiben, zueinander etwas Menschliches verspüren: „Es ist wie eine Entschuldigung voreinander, dass man noch da ist und nicht bei den vielen anderen in der Tiefe." (WBS, S. 413) Mit dem Ablauf der Zeit wächst auch die Kluft zwischen den Soldaten und Offizieren, die während des Krieges erst ihr Patent erhalten (Cron, H., [bearb.] Ein evangelischer 
Student, am 27.12.1916, Der Archiv des Deutschen Studentendienstes, Potsdam 1926, Mskrpt, S. 47, in: FA, S. 87). Es kommt auch zu Erneuerung der innerdeutschen Spannungen zwischen Bayern und Preußen, was sogar zum Blutvergießen führt (Bericht eines höheren Feldpolizeibeamten, dem Bayerischen Kriegsminister Kreis am 31.08.1916 vom stellv. preußischen Kriegsminister Franz Gustav v. Wandel übersandt, BHSta\IV. Mkr. 2335, in: FA, S. 53).

Die Einwirkung von oben erwähnten Faktoren bringt bedeutende Lockerung der Disziplin mit sich, so z.B. reagiert man schon im September (aber nicht beim Gefecht) auf die Zurufe des Offiziers nicht (vgl., KAK, S. 87), oder entgegnet die Worte eines Generals auf eine vulgäre Weise (vgl., O., S. 176) und im anderen Fall beteiligen sich selbst die Offiziere 1915 an einer Verbrüderung (Feldpostbriefe, Leutnant Fischer aus dem Weltkrieg 1915\1916, Brief vom 7.12.1915, BA\MA Freiburg, MSg 2\198, in: FA, S. 109)

Es gibt auch eine andere, parallele Wirklichkeit, die auch zu gleicher Zeit in den gleichwertigen Quellen erscheint. In dieser Wirklichkeit sind die Soldaten nicht müde, nicht verwildert, sondern heiter (vgl., FO, S. 39, 49, 54). Artilleriefeuer wirkt in dieser Welt kaum, stört sogar beim Lesen nicht (vgl., WB, S. 189; WBS, S. 185; BLF, S. 91) Die Gasangriffe werden „zur Sommerfrische“ (Grün, A.H, am 27.06.1916, KF, II, S. 12). Man nimmt vermutlich eigene Verstümmelung nicht ernst: „Und das eine Bein ist weg? Aber das andere behalte ich“ (VD, S. 33). Man baut phantastischste Bilder der Verharmlosung des Todes, den man sogar beneidet (vgl., WB, S. 174; Auszug aus einem Brief des Majors T., KF III, S. 27). Tote deutsche Soldaten sehen bei diesen Quellen ästhetisch aus, egal, ob noch unbegraben (vgl., FO, S. 112; 5 Monate, S. 107-108) oder in ,pietätvoll gezierten“ Soldatengräbern (LG, S. 34). Damit sind logischerweise Wunschbilder verbunden, die nicht nur von übereifrigen Etappesoldaten oder Journalisten erdacht sind, die „einen selbstgezähmten Engländer aus London mitbringen“ (Briefe von unsern braven Blaujacken, in Hamburger Fremdenblatt, nach: Herz, S. 32-33) wollen. Auch dreiste Lügen findet man neben wahren Berichten: „Wir lassen eine Bombe sausen und die Batterien da unten verwandeln sich in berstende Erzfetzen." (JW, S. 58), oder man beschreibt die Soldaten, die im Handgemenge singen (vgl., BLF, am 11.04.1915, S. 151), was schon aus physiologischen Gründen unmöglich ist.

Der einzige Feind, der in zwei Rollen - als Soldat und Zivilist - auftritt, sind die Franzosen. ${ }^{6}$ Man sieht aber schon am Anfang die vorurteilsvollen Meinungen, die wohl längst im Bewusstsein des Deutschen existierten: „3 Franzosen 10Pf, ein Dutzend billiger; Sitzungszimmer für die Kommission zur Eingemeindung Frankreichs..." (KAK, S. 18). Die Bilder von den ersten sind ebenso verschieden als von den zweiten, die Urteile sind nie eindeutig, die negativen überwiegen jedoch.

Manche französische Soldaten sind militärisch mehr erfahren als die deutschen, denn die Deutschen schießen über die Brustwehr, was gefährlich ist, die

\footnotetext{
${ }^{6}$ An der Ostfront sind die zivile „Russen“ eigentlich Polen.
} 
Franzosen dagegen - über die Schiesscharten ( vgl., KAK, S. 172), was eindeutig vernünftiger ist. Aber daneben gibt es sehr negative „typisch französische“ Handlungsweisen: sie gebrauchen Dum-Dum-Geschosse WKR, Nr. 7, vom 2.09.1914) und brechen dadurch die Kriegsregeln, schießen ,wild und sinnlos, um die Nerven zu beruhigen" (W, S. 82-83), sind feige (vgl., KAK, S. 188; K, S. 91) und kämpfen betrunken (vgl., KAK, S. 218). Man glaubt, dass diese unordentlich gekleidete Masse (vgl., DK, vom 26.09.1914, zitiert nach „Neckar-Echo“, S. 205), von den Offizieren, die den eigenen Soldaten fremd sind (Vgl., LuP. S. 201-202) mit Waffengewalt in den Kampf getrieben worden ist (vgl., KF III, vom 25.02.1915, S. 47), bis $2 / 3$ von ihrem Bestand fällt (vgl., PE, S. 286). Man bemerkt wohl deshalb bei den Franzosen kaum Angriffsgeist (vgl., KAK, S. 81). Addiert man dazu ihre Heidenangst vor Verkrüppelung (vgl., Corday, Michel, PE, S. 99), dann versteht man leichter ihr vermeintlicher minimaler Widerstand und Neigung zur Gefangennahme (vgl., Passow, A., vom 15.09.1915, KF II, S. 75). Die Neigung zum Kapitulieren ist mehrmals erwähnt, die gefangenen Franzosen sind fröhlich, lachen, scherzen und zeigen die Bilder von ihren Familien (vgl., KAK, S. 57, 95; WBS, S. 56). Die Sentimentalität bedeutet jedoch nicht, dass Franzosen hochmoralisch sind, denn selbst in dem ersten Graben haben sie die Frauen mit dabei (Aus einem Schützengraben, vom 6.10.1914, nach „Fränkischer Kurier“ in DK4, S. 119). Deshalb entstehen auch leichtfertige Urteile: „Die französische Armee war vollkommen geschlagen und demoralisiert..." (Fink von Finkenstein, Konrad, Gesicht der Marneschlacht, in: Flammende, S. 32) Es gibt überhaupt zahlreiche Versuche, deutsche Überlegenheit nicht nur im militärischen Bereich zu demonstrieren, sondern die allgemeine kulturelle Überlegenheit zu unterstreichen. Die Franzosen sind generell schmutzig (vgl., T, vom 20.09.1914, S. 39), das ganze Land ist in jeder Hinsicht verfallen (vgl., KAK, S. 161), die Technik veraltet, den deutschen Maschinen gegenüber ,wie ein strammer, verlässlicher deutscher Soldat neben einem wehleidigen Ohlala..." (LG, S. 57). Gleichzeitig sind Franzosen eindeutig unmenschlich, was ihr Verhalten den eigenen Soldaten gegenüber beweist. Sie sammeln die eigenen Gefallenen nicht (vgl., LG, S. 34) und werfen die Leichen aus dem Graben (vgl., ebenda, S. 37), mehr noch, falls die deutsche Soldaten französische Verwundete zu retten versuchen, scheiterten diese Versuche im Sperrfeuer (vgl., BLF, am 5.11.1914, S. 24). Gruselgeschichten begleiten manchmal Bilder des Gegners, besonders in der Bearbeitung der Journalisten (vgl., DK IV, am 1.10.1914, S. 215). Auch die Soldaten bemerken bei Franzosen die Elemente des psychologischen Krieges, wie Spottkarten auf deutsche Soldaten (vgl., LuP, S. 159) und französische Flugblätter für eigene Soldaten, die allerlei Hetzelemente ${ }^{7}$ in die Soldatenmasse bringen (vgl., KAK II, S. 87; London und Paris im Krieg, S. 178-179). Soldaten im Feld sind anderer Meinung und be-

\footnotetext{
${ }^{7}$ Man wirft den Deutschen vor, „französische Geiseln unter unserem Feuer marschieren zu lassen.“ (BS, am 13.09.1914, S. 40)
} 
trachten französische Soldaten als Mitleidende (vgl., O, S. 134, 164), als tapfere, obwohl sorglose Kämpfer (vgl., KAK, S. 145), die auch an der Front kultiviertes Leben führen: „Im Wald steht eine Lesehalle, auf den Wiesen sieht man sie Fußball spielen, ein kleines Bassin ist zum Baden geschaffen.“ (JW, S. 33) Das Gesagte erklärt auch wohl nicht die weitverbreiteten, aber jedoch signifikanten Tatsachen. Treiben die Befehlshaber die Gegner nicht in den Kampf, ist eine friedliche Koexistenz und Unterhaltung möglich (vgl., Töns, Franz, am 13.12.1914 an seine Mutter, Privbes., in: FA, S. 111). Selbst die Warnung vor den französischen Sprengungen kommen vor (vgl., Eine Verbrüderung mit den Franzosen bei Givenchy, Oktober 1915, verfasst von Otto Weber aus dem Veteranen- und Kriegsverein Buchloe, BHst A/IV, HS 1984, in: FA, S. 111). Und obwohl die ,journalistisch geprüften“ Dokumente Angst säen und vor der Gefangenschaft warnen: „Was wäre mein Los in Frankreich gewesen? Beschimpft, gemordet hätten sie mich." (Herz, S. 79, nach: „Leipziger Neuesten Nachrichten“), so ist eine durchschnittliche Mutter anderer Meinung: „Mein Sohn hat einige Fluchtversuche unternommen.“ (Die Mutter des Gefangenen B.Poll, in: KFVI, am 15.05.1918, S. 30). Die letzte Tatsache widerspricht völlig dem Bild des brutalen Franzosen, denn mehrere Fluchtversuche können auch während des Krieges mit dem Tod enden.

Weil die deutschen Frontsoldaten eine längere Zeit mit französischen Zivilisten koexistieren müssen, lohnt es sich, auch darüber etwas zu sagen. Und obwohl sogar französische Frauen gegen die deutschen Truppen unmenschlich vorgehen (vgl., T., am 6.09.1914, S. 32), sind die Beziehungen eher gut als schlecht, man versorgt Franzosen mit Proviant (vgl., An Maas und Aisne, nach „Berliner Local Anzeiger", in: DH, S. 118) und die Franzosen servieren deutschen Soldaten Kaffee und Kuchen (vgl., Gesicht der Marneschlacht, in: Flammende, S. 33).Die Beurteilung der menschlichen Qualität der französischen Zivilisten fällt eher negativ aus, weil die vertrauensunwürdig sind. Ein französischer Lehrer nennt die Deutschen vor ihrem Ankommen „Barbaren“ und nach ihrem Ankommen - „Freunde“ (LG, S. 52). Sogar im letzten Kriegsjahr verspotten die französischen Zivilisten die gefangengenommenen Alliierten, was die Deutschen verwundert: „Ist das der große Massentrieb, der sich zum Sieger schlägt und den Besiegten verachtet?" (TBR., am 27.03.1918, S. 78) Dabei versteht man auch die Schwierigkeiten der interkulturellen Kommunikation: „,... auch die höchste kulturelle Verfeinerung hilft nicht, einen Franzosen völlig zu verstehen und nun gar einen Russen“"(KAK II, S. 120). ${ }^{8}$

${ }^{8}$ Die Meinung der Soldaten formen sind zwiespältig: „Von einem Volksantagonismus gegen die Russen war keine Rede und konnte keine Rede sein.“ und gleich danach: „'Der Russe hasst den Deutschen, weil Deutschland das Nachbarland ist, das zum Vergleich reizt und dessen überlegene Zivilisation seinen Barbarenstolz demütigt, " - so urteilte im Jahre 1905 ein außenstehender objektiver Beobachter, wie der belgische Gesandte Baron Greindl in Berlin." Oncken, H., Deutschland vor dem Ausbruch des Krieges, in: DGK, S. 7. Hätte der Baron etwas in Russland gelesen, dann hätte er wohl etwas vernünftiger formuliert, denn für $99 \%$ der Bewohner Russlands damals lag Deutschland nirgendwo und war wohl nicht näher als der Mond oder andere Planeten. 
Das Russenbild ist im Voraus bei den Truppen voll negativer Emotionen, die einen spezifischen Krieg vorbereiten. Manche ziehen in den Graben ideologisch vorbereitet: „Unser alter Bebel hat gesagt, gegen Russland trüge er am liebsten noch selbst die Flinte." (Heimwärts - Reise durch die Mobilmachung, in Herz, S. 13). ${ }^{9}$ Es entstehen schnell absurdeste Legenden: „In Odessa - Aufstand, Straßenkämpfe, Truppen verräterisch, ein Panzerkreuzer bombardiert die Stadt.“(WKR, NR. 5, am 27.08.1914, unpaginiert). Dazu kommt auch eine ziemlich große kulturelle Ignoranz, die bestimmt auch auf andere Beurteilungen einwirkt: das Land im Osten bewohnt ein primitives Volk - „Panjes“ (Otto, F.B., am 29.05.1916, KF II, S. 18), es erscheint ein russischer Major (Vgl., Gefangen und wieder befreit, DK VI, S. 125, nach ,Essener Arbeitszeitung ${ }^{\circ}$ ), obwohl es zur Zeit in der russischen Armee keinen solchen Rang gibt, es erscheinen bewaffnet im Bett schlafende Kirgisen (Vgl., JK, S. 45), die in der Wirklichkeit vom militärischen Dienst in Russischen Reich befreit sind. Manche Soldaten sehen und beurteilen diese Problematik der Volkshetze und Haßerziehung: „Überall lagen dort die albernen Witzkarten auf die Russen umher, die unser nicht würdig sind..." (5 Monate, am 20.10.1014, S. 74) Diese Feindbilder sind nicht so selten: „Dem Feinde zu Ehre sei es gesagt: zuleide haben sie keinem was getan.“ (Unter Feuer und Schwert, DK II, am 25.09.1914, S. 47, nach ,Vorwärts ${ }^{6}$ Berlin). ${ }^{10}$ Manchmal erscheint die positive Bewertung neben dem politisierten negativen Urteil: , 'Mir hat ein russischer Offizier Milch gereicht, sagte ein angekommener Verwundeten. Mich zog ein Russe hinter einen schützenden Hügel', so versichert ein anderer." Und gleich danach spricht der Schreibende über „Halbbarbaren“: „Das sind die eigentlichen Stützen des Zarismus, dieser Geißel Europas..." (LuP, am 25.08.1914). Man bemerkt selbst in einer Quelle nicht, dass die Urteile einander widersprechen, denn mal sind Brand und Einbruch die „Unterhaltung" der russischen Soldaten (Vgl., VW, S. 75) und plötzlich bezahlen dieselben alles, was sie brauchen (Vgl., VW, S. 91). ${ }^{11}$

Erste Kämpfe verlaufen noch fast ritterlich, und als 3 russische Parlamentäre bei der Feste Boyen beschossen und verwundet werden, verspricht der deutsche Festungskommandant „die strengste Bestrafung“ der Schuldigen und Rückkehr

9 Oder: ,...gegen Russland zieht man freudiger in der Hoffnung, dieser Unkultur und dem Knutenregiment ein Ende zu bereiten." - In Tschenstochow, DK VI, S. 31, nach ,Berliner Tageblatt‘.

${ }_{10}$ Manchmal findet man eben bei Frontsoldaten viel positive Aussagen über den russischen Gegner: „Es würde viel geschwindelt, meinte er; bis zum vorigen Sonntag sei er in seiner Ortschaft geblieben, da seien die Russen eingezogen, und es sei nichts geschehen." - LuP, am 29.08.1914; „Erwachsene Kinder sagten mir: ,Die Russen waren nie frech...'“ - LuP, am 15.09.1914, S. 62; „Wie ich von den Frauen erfuhr, benahmen sich die Russen ihnen und den Kindern gegenüber ganz manierlich.“- LuP, am 8.11.1914, S. 124; „Die Russen sind für Bauern und Frauen harmlos.“ (H.C., am 7.12.1916, S. 185)

${ }^{11}$ Symptomatisch sind die Tatsache, dass die Neuigkeiten die über ,unbeschreiblichen Gräueltaten" berichten, vom Ausland stammen - z.B. DK II, S. 54, aus ,Düsseldorfer Zeitung'; S. 70 aus ,Post', Berlin, beide nach: ,Aftonbladet', Stockholm. 
der Verwundeten aus dem deutschen Lazarett. (Vgl., KD, am 14-27.08.1914, S. 2) Als Gegner bewertet man die Russen sehr unterschiedlich, mit Franzosen und Engländern unvergleichbar. Der russische Feind handelt im Allgemeinen mit Verachtung des fremden und eigenen Lebens (Vgl., LuP, 8.09.1914, S. 48; FO, S. 118), kämpft jedoch sichtbar schlechter, als Deutsche: „, Denn Drang nach vorwärts liegt im Blut und ist nicht erlernbar. Die Russen haben dagegen das Erlernbare gelernt - die Kunst der Verschanzung." (Die Kämpfe vom 10. und 11.Oktober,am 13.10.1914, DK VI, S. 150, nach ,Vossischer Zeitung'; auch vgl., FO, S. 117-118). Viel öfter sieht man aber andere Urteile über russische Fortifikationen, wo eigentlich alles nachlässig gebaut ist, und die Unterstände - eine hygienische Katastrophe (vgl., 5 Monate, am 19.10.1914, S. 71-72; MF, S. 159; PE, am 1.05.1915, S. 135). Man bestätigt eine sehr gute Qualität des russischen Artilleriefeuers (vgl., 5 Monate, S. 81), dagegen missachtet man andere Waffengattungen. Die Infanterie erträgt den Nahkampf nicht (vgl., Goes, G., Der kleine Spielmann, in: Flammende, S. 100-101), vermeidet womöglich die Kämpfe mit deutschen Einheiten (Vgl., WBS, S. 201) und kapitulieret sehr schnell bei einem Sturmangriff (vgl., Warum die Offensive abgebrochen wurde, DKG, S. 204-205, nach ,Frankfurter Zeitung'; Kampf und Vormarsch, DK X, S. 156, nach: ,Volksstimme', Magdeburg). Man unterstreicht meistens die gute Ausrüstung und Verpflegung russischer Soldaten (Vgl., 5 Monate, am 5.10.1914, S. 45; VW, S. 59; FO, S. 101) ${ }^{12}$, andere Meinungen sind selten (vgl., DEW, S. 90).

Russische Soldaten kämpfen tapfer und treu in zerschossenen Graben (Vgl., VW, S. 45; A.M., S. 179) und die Sibirier ,schlagen alle Angriffe ab und ziehen sich erst im Schutze der Nacht [...] zurück."(WBS, S. 99). ${ }^{13}$ Jedoch fehlt ihnen entsprechende Kriegsschulung, dass sie sogar eigene Flieger herabschießen (vgl., 5 Monate, S. 85), ihre technische Ausrüstung zeugt von Knappheit des Kriegsmaterials, als sie in Minen Ladungen aus alten Nägeln verwenden (vgl., J.W., S. 34). Im zweiten Kriegsjahr, als die russische Armee schon keine Offiziere aus der Friedenszeit hat, macht man die Bemerkung, dass die Offiziere versagen (Müller, am 4.10.1915, KF V, S. 40). Die letzten begleiten im Graben (wie bei Franzosen) „galante Damen“ (5 Monate, S. 14), was sicherlich der kriegerischen Beschäftigung schadet.

Manchmal ist die Rede über die Zwänge, die die russische Seite gebraucht, um die Einheiten zum Kampf zu zwingen. Mal nötigt der Nachgreifende den Vorgreifenden (vgl., Osterschlacht in Karpaten, DGK, S. 125), mal kommt der Offizier mit geladenen Pistolen, was „ein paar recht intelligente Polen“ bestätigt

${ }^{12}$ Und diese Soldaten trinken nicht Wodka, sondern Tee aus ihren Feldflaschen, was den Deutschen zutiefst verwundert - Vgl., LuP, am 30.10.1914, S. 113.

${ }^{13}$ Deshalb bemerkt man, dass trotz Tannenberg ist der russische Gegner noch gefährlich (vgl., am 3.10.1914, S. 37) und deutsche Verluste „unverhältnismäßig groß“ sind (Am Wislok, DK X, S. 158, nach: ,Straßburger Post'). 
(5 Monate, S. 81-82), oder man verwendet die Artillerie ,als Peitsche“" (ebenda, S. 192). ${ }^{14}$ Man macht kein Geheimnis aus der Tatsache, dass die deutsche Seite Haager und Genfer Kriegsregelungen in Bezug auf Russen nicht besonders beachtet: im Stroh versteckte russische Soldaten ersticht man ohne Vorwarnungen und Aufforderung zum Ergeben (vgl., 5 Monate, S. 105), man tötet Unverwundeten die sich unter Verwundeten verkriechen (vgl., Die Schlacht an den masurischen Seen, DK, S. 173) oder man gibt kein Pardon den kapitulierenden russischen Artilleristen (vgl., FA, S. 57, nach: ,Berliner Tageblatt‘ vom 4.04.1916). Den Russen gegenüber macht man jedoch keine solche Vorwürfe, noch mehr, es gelingt gemeinsam mit Russen die Gefallenen zu begraben (vgl., MK, am 1.03.1915, S. 71), und fast friedlich leben: „Wenn wir die Russen in Ruhe lassen, tun sie uns auch nichts..." (KF I, am 26.07.1915, S. 46).

Deutsche Gefangenschaft betrachten die Russen, der deutschen Meinung nach, schon vor der Ankunft an die Front als eine fröhliche Perspektive. Sie bitten sogar deutsche Kriegsgefangene um einen Passierschein, „Propusk“, um nach Fahnenflucht gut behandelt zu werden (vgl., v. Schmidt, F., Als Gefangene nach Sibirien, in: Flammende, S. 154-156). Solche Überläufer gibt es schon am Anfang des Krieges (vgl., MK, am 25.02.1915, S. 68), neben den anderen, die einfach nicht schießen wollen (vgl., LuP, am 6.11.1914, S. 121), oder ihr Leben um jeden Preis retten möchten, was deutsche Soldaten vermutlich zum Lachen bringt (vgl., FO, S. 170). Andere wollen „hochstehende deutsche Kultur lernen“ (WF, S. 30), bei anderen noch liegt aber "Trotz und Scham in ihrem Ausdruck“ (5 Monate, 10.09.1914, S. 24). Die Russen benehmen sich in der Gefangenschaft ihrer Herkunft nach: „, Man konnte feststellen, dass die russischen Offiziere zunächst um Zigaretten und Briefpapier baten, die Mannschaften um Brot, die jüdischen Gefangenen fragten aber als erste Frage: 'Wie hoch steht hier eigentlich der Rubel im Kurs, Herr Leutnant?'“ (5 Monate, am 4.12.1914, S. 127). Das ist eigentlich dank der anderer Quelle verständlich, denn zuerst nimmt man Russen Brot, Zucker, Tee und Zigaretten ab (Vgl., DK VI, S. 111). Die alle - Überläufer und treue Soldaten erwartet nichts beneidenswertes, man behandelt sie wie Tiere, gibt Schweinfutter und erzielt eine Sterblichkeitsrate um 39\% (Vgl., T., im Juli 1918, S. 256), höher als an der Front.

Es gibt noch eine andere Sorte von Aussagen, die identisch mit der NS-Propaganda sind und einem einzigen Ziel dienen - den Feind zu entmenschlichen, ihn zum Tier, zum Untermensch zu machen. Das beginnt ziemlich harmlos, bei Darstellungen, dass die Russen an der Front dreckige, zerrissene Uniformen tragen (Vgl., Die Feuertaufe bei Stalluponen, in: DK II, am 17.08.1914, S. 101), sind schmutzig und verlaust (Vgl., T, am 20.09.1914; M.M., am 9.12.1914, S. 147)

${ }^{14}$ Auch die schon erwähnten Phantasieelemente erscheinen wieder als „Finnländer“ (5 Monate, S. 82), was eigentlich unmöglich ist, denn die Bewohner Finnlands waren in Russischen Kaiserreich vom Kriegsdienst frei. 
und riechen dazu unsagbar (Vgl., 5 Monate, am 7.09.1914, S. 13). Die Einseitigkeit solchen Behauptungen über das Frontleben entbehrt jeglicher Kritik, denn man versucht dem Feind etwas zuzuschreiben, was zu beiderseitigen Frontalltag gehört. Eine Unmenge von identifikativen Merkmalen, die den Feind zusätzlich aus der Gemeinschaft der Europäer verdrängen sollen, folgt: „Asiatenaugen“ (FO, S. 32), „Sibirjaken, wilde Asiaten“ (J.W., S. 27), „Söhne Asiens“ (Brettner, am 21.03.1915, in: KF IV, S.10), „wilde Sibirier“(LuP, am 5.10.1914, S. 93), aus welchen die ,asiatische Barbarei“ ausbricht (BLF, am 6.06.1915, S. 207). Es gibt eine wahre Fundgrube, die den Gegner entwerten: „,bestialische“ (DK II, S. 1916, nach: ,Chemnitzer Volksstimme“) „Horden aus Osten“ (A.M., S. 205) wüten in Ostpreußen auf solche Weise, dass die Autoren dieses Land als „Massengrab“ (LuP, S. 65) bezeichnen und die Kriegshandlungen als „eine einzige ungeheurere Anklage gegen die russische Regierung und gegen die russische Armee." (Brachmann, A., Ostpreußen, DGK, S. 162). Danach kommen primitive Beleidigungen, die als Epiteta an dem russischen Feind kleben: „Räuber und Mordgesellen“ (5 Monate, am 14.09.1914, S. 28), ,feige Mordgesellen“ (Zoozmann, R., Ein Julitag in Berlin, in: Herz, S. 18-19), „Hornochsen und Lümmels“ und „blödsinnige Russenbande“ (MK, 17.02.1915, S. 104) oder „russische Saubengel“(Herz, S. 60) nach ,Berliner Tageblatt', die dazu noch „dreckige Mistfinkenhöhlen“ (5 Monate, S. 146) bewohnen.

Gefangen sind die Russen „dumpf in ihr Schicksal ergeben“(WBS, S. 99), „gehorchen sklavisch“ (LuP, am 15.09.1914, S. 54) und sind, wie es schon oben erwähnt wurde, eigentlich die Untermenschen: „Ich versuche, einen Geistesfunken bei ihnen zu entdecken - vergeblich. Völlig unwissend sind sie, unaufgeklärt. [...] Selbsterhaltungstrieb, blinder Instinkt.“ (ML, S. 28-29). Solche Untermenschen tötet man dann mit besonderer, lustvoller Freude: „Wann dö russischen Schädelen so knirschen, däs ischt ein eigentümliches Geräusch.“ (FO, S. 54)

Die Unterschiede reichen so weit, dass selbst tote Feinde anders sind, tierisch, weil „gorillahaft angeschwollen“"(Jaeger, Fr., am 31.08.1914 in: KF IV, S. 21) und haben nur ,entstellte und verwüstete Gesichter" (FO, S. 112). Nach solchen Enthüllungen führt die Denkweise zu einer endgültigen Feststellung - der russische Feind ist überhaupt kein Mensch sondern ein Tier (vgl., Die Straße des Todes, Finkenstein, v.F, Flammende, S. 102-109; WBS, S. 296-297; J.W, S. 75). Gefangen ruft man sie „zum Fressen“ (vgl., 5 Monate, S. 78) und „es war nur schwer zu unterscheiden, wo der menschliche Haarwuchs sich von der tierischen Pelzmütze trennte" (J.K, S. 52).

Solchen Soldaten kann man alles zutrauen - Pogrome (Vgl., Vom Ruhm des deutschen Mannes, in: DK X, am 30.06.1915, S. 254, nach: ,Neue Preußische Zeitung-Kreuzzeitung') oder sogar nie vorkommende - aber mögliche - Verbrechen: „.. wenn nicht inzwischen die deutschen Truppen eingerückt waren, hätte Allenstein die andere Seite des russischen Soldaten kennenlernen können.“ (5 Monate, S. 14) Logischerweise ist der deutsche Sieg über russischen Feind so 
gut wie garantiert, und man bemerkt wohlwollend, „wie Tolstois hölzerner Bau krachend zusammensank, wie stolz der deutsche Übermensch sein Haupt erhob!““ (BLF, am 30.10.1914, S. 16-17)

Die Bilder von Engländern sind ganz anders, vielleicht auch deshalb, weil man sie als Verbündete im zukünftigen Krieg gegen Frankreich und Russland betrachtete. Diese Meinung war nicht unbegründet, denn die alte Feindschaft zwischen diesen Ländern fand sogar reiche Widerspiegelung in der Literatur. ${ }^{16}$ (M.F., S. 55-56) Negative, erniedrigende Formulierungen und Taten den Engländern gegenüber sind sehr selten, irgendwo am Rande erschein „Lumpenhund“ ( T, am 9.08.1914, S. 14) oder es werden ausgestopfte Soldaten in den Unformen der englischen Leibgrenadiere an Geschützwagen angenagelt (ebenda, am 2.09.1914, S. 29). Die englische Kampfweise wird ohne Kommentare dargestellt, egal, ob es um Kriegsverbrechen geht, als die Engländer schwimmende deutsche Schiffsbrüchige im Wasser erschießen (vgl., WBS, S. 121), Dum-Dum-Geschosse gebrauchen (WKR, Nr. 7, am 2.09.1914, unpag.,), oder ihre Toten nicht begraben (PE, S. 349; VD, S. 53, 110). Und als sie heimtückisch handeln und kapitulierend Handgranaten werfen und in deutschen Uniformen kämpfen, so schreibt man diese Taten den Kolonialtruppen zu. (vgl., VD, S. 124-125)

Üblicherweise werden die Engländer aber bewundert, meistens eben wegen ihrer Kampfweise, als „harte Kämpfer" (Goes, G., Der Laufer von Gillemont, in: Flammende, S. 263-265), als Soldaten, die sich ,in ihren Besitz verbeißen und über ihr Eigentum die Löwenpranken halten." (Feuer, S. 61). Den erfolgreichen MG-Schützen, der mehrere Dutzend Deutsche tötete, beurteilt man als einen „richtigen Mann am richtigen Fleck“ (Feuer, S. 154), englische Soldaten „wehrten sich wirklich glänzend“ (Die erste deutsche Durchbruch 1918-Der Beginn der Infanterieschlacht, in: Front, S. 74, nach Junger. E. In Stahlgewittern). Man hat bestimmt mit diesen kein Mitleid im Krieg (vgl., Witkop, Ph., Der Fähnrich, in DGK, S. 74), aber diese Feinde wissen vermutlich besser als alle andere, wofür sie kämpfen (vgl., Aus Kriegsakten Reichskanzlei 1914, 2398/7-8, Bl. 49, 50/51, in: FA, vom 18.08.1916) Und im Hintergrund dieser Urteile sind ja mehrere Tausende deutsche Soldaten, die von dem musterhaften Feind umgebracht worden sind. Man bewundert eine „Gummi-Feld-Badewanne“ (LuP, S. 39), ihre Haltung, ihre Ausrüstung und Entschlossenheit, sogar ihren Humanismus (T., am. 20.09.1914, S. 39; am 13.10.1914, S. 45; im Juni 1918, S. 245). Manchmal fehlen den Autoren die Worte, um ihre Begeisterung sogar für gefangene Engländer zu beschreiben,

${ }^{15}$ Recht seltsam wirkt in dieser Flut von brutalsten Formulierungen die Bemerkung, dass russische Damen ,in einigen Städten gefangenen Offizieren Blumen und Obst“(A.M., S. 195) bringen, also - dieselben Damen, die wie alle Russinnen zugleich Philosophinnen und Tiere sind (Vgl., A., S. 206)

${ }^{16}$ Battle of Dorking, Battle of Bologne, How John Bull lost London, The great naval war, The capture of London. Diese öffentliche Meinung existierte jedoch nur in Deutschland, denn in England dachte man anders: „Wenn Deutschland morgen aus der Welt vertilgt würde, so gäbe es übermorgen keinen Engländer in der Welt, der nicht umso reicher sein würde..." (Wilhelm II, am 11.09.1897, S. 28) 
man will in dem Feind nur das Positive sehen, denn, ,in diesen Gestalten prägt sich eine gesunde, tüchtige, ernste Rasse aus, von der die Welt noch viel zu erwarten hat" (BLF, am 24.03.1915, S. 138), die auch in der Gefangenschaft „ganz ungebeugt" (EF, am 17.06.1916, S. 176) ${ }^{17}$ bleibt.

Die Briten sind so artig, dass ihre Karikaturen über die deutschen Soldaten „harmlos" sind (LuP, S. 35), was der Wirklichkeit nicht entspricht. Aus der Bewunderung für den Feind lassen sich leicht „friedliche Verhältnisse“ (Aldag, K., am 3.01.1915, in: KS, S. 31) und die gute Atmosphäre mit Engländern erklären (vgl.,v. Rohden, G., in: KS, S. 121), und das auf verschiedenen Frontabschnitten. Sogar am Ende des Kriegs, als die allgemeine Verwilderung überall herrscht, beschreibt man pietätvoll einen Deutschen, der auf seinem Rücken sorgsam einen Tommy trägt. (TBR, am 25.03.1918, S. 62). Nur selten kommen andere Bilder vor, die eher zu der Endphase des Krieges gehören. Dann erscheinen die flachen englischen Stahlhelme als „ekelhaft“ (Zöberlein, H., Der Tod von Ypern-Flandernschlacht 1917, in: die Front, S. 32) und die Einrichtung der englischen Hauptkampflinie „erbärmlich schlecht“ (TBR, am 27.03.1918, S. 74).

$\mathrm{Zu}$ erwähnen ist auch die englische Brutalität den Franzosen gegenüber, die deutsche Beobachter in Erstaunen versetzt. So zerschießt die englische Artillerie im Frontgebiet französische Städte samt Bewohner (vgl, VD, S. 120) Auch in der Gefangenschaft vermeidet der Engländer Kontakte mit Verbündeten, ,die er im Grunde seines Herzens für Geschöpfe minderer Art hielt.“ (WB, S. 89) Sogar am Ende des Krieges, als die Zustände in der deutschen Armee wegen fehlender Menschenreserven schon fast katastrophal waren, kommt es zwischen gefangenen Engländern und Franzosen aus besten Einheiten, zu wüsten Szenen (vgl., TBR, am 25.04.1918, S. 152-153).

Als Schlussfolgerung kann man am besten die Aussage von Anatol France aus dem Jahre 1917 zitieren, die alle dargestellten Bilder summiert: „Nicht nur, dass der Krieg diese furchtbaren Leiden verursacht; er verwandelt alle, die nicht verrückt werden, in Idioten.“ (PE, S. 670)

\section{LITERATURVERZEICHNIS}

Beumelburg W. (1929), Sperrfeuer, Oldenburg.

Beumelburg W. (1933), Flandern, Oldenburg.

Blond G. (1989), Schlacht von Verdun, „Der Spiegel”, 33.

Blücher von Wahlstatt G.L. (1924), Fürstin, Tagebuch, München.

Born D. (1915), Meine Erlebnisse in der Russenzeit, Königsberg i. Pr., Koch.

Brandt R. (1915), Fünf Monate an der Ostfront, Fleischel, Berlin.

Carossa H. (1924), Rumänisches Tagebuch, Insel-Verlag, Leipzig.

${ }^{17}$ Die Behandlung des Begriffs „Rasse“ ist bei allen Quellen aus dieser Zeit immer nicht wissenschaftlich sondern politisiert umgangssprachlich. 
Chevillon A. (Hrsg.) (1918), Briefe eines Soldaten, Zürich.

Dahms P. (1915), Wir von der Infanterie, Berlin.

Delbrück J. (Hrsg.) (1915), Der deutsche Krieg in Feldpostbriefen: der Feldzug Polen, Bd. 6, München.

Delbrück J. (Hrsg.) (1915), Der deutsche Krieg in Feldpostbriefen: Hindenburg und Tannenberg, Bd. 2, München.

Delbrück J. (Hrsg.) (1917), Der deutsche Krieg in Feldpostbriefen: Die Durchbruchschlacht in Westgalizien, Bd. 10, München.

Delbrück J., Imhoff K. (Hrsg.) (1915), Der deutsche Krieg in Feldpostbriefen: Um Longwy und Verdun, Bd. 4, München.

Deutsche Feldpostbriefe, Schilderungen und Bericht vom Völkerkrieg (1914), „Chemnitz”, 11-12, 14-29.

Deutsche Kriegs-Vaterlands und Soldatenlieder (1916), Bd. III, Berlin.

Düwell W. (1914), Kriegsberichte aus Ostpreußen und Russland, Berlin.

Ehrenberg H. (1915), Der Krieg und die Kunst, Kriegsvorträge der Universität Münster, Münster.

Engel E. (1916), 1914-1916: Ein Tagebuch, Berlin-Braunschweig-Hamburg.

Englund P. (2011), Schönheit und Schrecken. Eine Geschichte des Ersten Weltkrieges, erzählt in 19 Schicksalen, Berlin.

Exerzier-Reglement für die Infanterie vom 29 Mai 1906. Neuabdruck mit Einfügung der bis April 1913 ergangenen Änderungen (1913), Berlin.

Ferro M. (1988), Der Große Krieg 1914-1918, Frankfurt a.M.

Franke R. (1916), Die Werke westpreußischer Frauen an der Ostfront. Eine Reiseschilderung, Danzig. Ganghofer L. (1915), Die Front in Osten, Berlin-Wien.

Ganghofer L. (1915), Die stählerne Mauer, Berlin-Wien.

Gomoll W.C. (1916), Im Kampf gegen Russland, Leipzig.

Hardt F.B. (1917), Kulturdokument zum Weltkrieg, Bd. 1, München.

Hilfsverein Deutscher Frauen (Hrsg.) (1914-1915), Weltkrieg. Kriegs-Ruhmesblätter 1914-1918, Einzelausgaben: 1914: Nr. 2-5/7/10/ 12-13/20/22/26-27/33/37/39/42 // 1915: Nr. 50 / 55-56/ $61 / 75 / 79 / 81 / 87$.

Hirschfeld G., Krumeich G. (2013), Deutschland im Ersten Weltkrieg, Frankfurt a.M.

Hirschfeld G., Krumeich G., Langewiese D., Ullmann H.-P. (Hrsg.) (1997), Kriegserfahrungen. Studien zu Sozial- und Mentalitätsgeschichte des Ersten Weltkrieges, Essen.

Hirschfeld G., Krumeich G., Renz I. (Hrsg.) (1996), Keiner fühlt sich hier mehr als Mensch ..., Erlebnis und Wirkung des Ersten Weltkrieges, Essen.

Hochschild A. (2013), Der Große Krieg. Der Untergang des Alten Europa im Ersten Weltkrieg, Stuttgart.

Jacques N. (1915), London und Paris im Krieg - Erlebnisse auf Reisen durch England und Frankreich in Kriegszeit, Berlin

Jäckh E. (Hrsg.) (1916), Der große Krieg als Erlebnis und Erfahrung. Band 1/3, Das Er-lebnis, Gotha. Jünger E. (1929), Feuer und Blut, Hamburg, Wandsbeck.

Kern O. (Hrsg.) (1917), Kriegsbriefe eines deutschen Studenten, Halle.

Kokoschka O. (1976), Vom Erleben. In: E. Turnher (Hrsg.), Dichtung aus Österreich. 2. Teilband, Wien.

Konarska J. (1996), Wojna nad dworem, „Karta”, nr 19.

Krach O. (Hrsg.) (1915), Das deutsche Herz - Feldpostbriefe unserer Helden, Berlin.

Kriegs-Almanach, (1915), o.V., Insel Verlag, Leipzig.

Kutscher A. (1915), Kriegstagebuch. Namur - St.Quentin - Petit Morin - Reims - Winter-schlacht in der Champagne, (1916), Teil 2, Vogesenkämpfe, München.

Kübel J. (1915), Beim Landsturm. Feldbriefe, Berlin.

Langen A., Müller,G. (Hrsg.) (1937), Der deutsche Soldat. Briefe aus dem Weltkrieg, München. 
Liuevicius V.G. (2002), Kriegsland im Osten, Hamburg.

Lossberg F. von (1939), Meine Tätigkeit im Weltkriege. 1914-1918, Berlin.

Madelung A. (1915), Mein Kriegstagebuch, Berlin.

Mann G. (1980), 1914 und 1980, „Die Welt” 30.04.

Manz G. (Hrsg.) (1915), Von Flandern bis Polen. Feldpostbriefe der Täglichen Rundschau aus dem Weltkriege, Berlin.

Matthes E. (Hrsg.) (1915), Kriegs-Fahrt. Wandervogel-Feldbriefe, Leipzig.

Mein Kriegsfreiwilliger - Briefe und Tagebuchblätter eines in Russland gefallenen deut-schen Soldaten. Herausgegeben von seinem Vater, (1915), Bielefeld-Leipzig.

Merwin B. (1915), Mit den polnischen Legionen in den Karpaten. 1914, München-Berlin.

Michalka W. (Hrsg.) (1994), Der Erste Weltkrieg. Wirkung, Wahrnehmung, Analyse, München.

Michel R. (Hrsg.) (1917), Briefe eines Landsturmleutnants an Frauen, Berlin

Müller-Rüdersdorf W. (Hrsg.) (1915), Mit Herz und Hand. Ein Heldenbuch vom Weltkriege, München.

Münkler H. (1988), Die Schuldfrage spielt keine Rolle mehr, „Die Zeit” 9.12, 50.

Papen F. von (1952), Die Wahrheit eine Gasse, München.

Passow H. (Hrsg.) (1915-1919), Kriegserinnerungen und Feldpostbriefe von Corps Saxonia zu Jena, Hefte I-VI.

Pohlmann M., Potempa H., Vogel T. (Hrsg.) (2014), Der Erste Weltkrieg 1914-1918, München.

Renn L. (2014), Krieg, Berlin-Weimar.

Rosner K. (1916), Vor dem Drahtverhau. Bilder aus dem Grabenkrieg im Westen, Berlin.

Rosner K. (1918), Die große Frühlingsschlacht 1918. Tagebuchblätter, Berlin.

Schlicht F. von (Wolf Graf Baudissin) (1916), Unsere Feldgrauen. Erzählungen, Berlin.

Schmidt E. (1915), Mit meiner Feldkompagnie bis an die Marne, Berlin.

Schwering J. (1914), Die literarische Fremdherrschaft in Deutschland, Kriegsvorträge der Universität Münster, Münster.

Sonnenthal H. von, Sonnenthal-Scherer M. (1918), Ein Frauenschicksal im Kriege, Berlin-Wien

Sturm H. (1915), Erlebnisse eines Kriegsfreiwilligen mit Nutzanwendungen für die deutsche Jugend, Leipzig-Berlin.

Tietz L. (1920), Ein Jahr Weltkrieg. August 1914 - August 1915, Düsseldorf 1915.

Ulitz A. (1920), Ararat, München.

Ulrich B., Vogel J., Ziemann B. (Hrsg.) (2001), Untertan im Uniform. Militär und Militarismus im Kaiserreich 1871-1914. Quellen und Dokumente, Frankfurt a.M.

Ulrich B., Zimmermann B. (Hrsg.) (2008), Frontalltag im Ersten Weltkrieg, Essen.

Unruh F. von (1919), Opfergang, Berlin.

Unsere feldgrauen Helden. Aus Tagebüchern und Briefen, (1915) Bd. I-II, Heymann, Robert (Bearb.), Leipzig.

Unsere Feldgrauen! Marsch und Lagerlieder. Textbuch, (1915), o.V., Leipzig.

Usadel G. (Hrsg.) (1936), Die Front des grauen Stahlhelms, Leipzig-Berlin.

Vollmer H. (Hrsg.) (1993), „In roten Schuhen tanzt die Sonne sich zum Tod“ - Lyrik expressionistischer Frauen, Zürich

Wachtfeuer - Künstlerblätter zum Krieg 1914/15, Wirtschaftlicher Verband bildender Künstler, Berlin. Nr. 23, 30, 31, 33, 39, 45, 157, 206, 216.

Walther K.A. (Hrsg.) (1933), Das Langemarkbuch der deutschen Studentenschaft. Im Auftrage der deutschen Studentenschaft, Leipzig.

Wegener G. (1915), Der Wall von Eisen und Feuer. Ein Jahr an der Westfront, Leipzig.

Wertheimer F. (1915), Von der Weichsel bis zum Dnjestr, Stuttgart-Berlin.

Wilhelm II (1921), Vergleichende Geschichtstabellen von 1878 bis zum Kriegsausbruch 1914, Leipzig.

Wir von der Infanterie. Tagebuchblätter eines bayerischen Infanteristen aus fünfjähriger Front und Lazarettzeit (1929), Lehmann, Friedrich, München. 
Witkop P. (Hrsg.) (1928), Kriegsbriefe gefallenen Studenten, München.

Zank W. (1989), Jetzt oder nie, „Die Zeit” 7.07.

Zedlitz-Trützschler R. (1924), Zwölf Jahre am deutschen Kaiserhof, Deutsche Verlags-Anstalt, Stuttgart-Berlin-Leipzig.

„Zeit-Echo” (1915-1916), 1-15.

Zipfel E. (Hrsg.) (1935), Flammende Fronten. Eindrücke und Erlebnisse aus dem Weltkrieg, Sulzbach in der Oberpfalz, o.J.

Anatol Kapphengst

\section{THE FRONT-LINE SOLDIER SEES HIMSELF AND THE ENEMIES - A BRIEF OUTLINE}

(Summary)

The presented article is an attempt to describe in a synthetic way an own vision of a German front-line soldier of the First World War. It also tries to show an image of some enemies - the French, the British and the Russians.

The attempt was made on the basis of recollection material and literary forms created either during or soon after the war.

The conclusion is unequivocal - the more contact the authors of the texts had with the opponent, the more balanced image of the enemy they presented in their texts and the less were they prone to overestimate the German soldier.

Yet the press material contained a lot of exaggeration. The authors created a haughty and sometimes even superhuman image of the German soldier. At the same time the image of the enemy was inhuman and deserving contempt.

Keywords: Image of war, role of propaganda, opinion of soldiers, personal image, image of enemies, national differences, dictionary of war 